\title{
Diretrizes para o ensino na Atenção Primária à Saúde na graduação em Medicina
}

\author{
Guidelines for Primary Health Care teaching in undergraduate medical \\ education \\ Estándares para la enseñanza en la Atención Primaria de Salud en el \\ pregrado médico
}

Marcelo Marcos Piva Demarzo', Rodrigo Cariri Chalegre de Almeida², João José Neves Marins², Thiago Gomes da Trindade ${ }^{1}$, Maria Inez Padula Anderson ${ }^{1}$, Airton Tetelbom Stein², Fabiano Gonçalves Guimarães ${ }^{1}$, Felipe Proenço de Oliveira², Fernanda Plesmann de Carvalho ${ }^{1}$, Flavio Dias Silva ${ }^{1}$, Francisco Arsego de Oliveira², Gustavo Tenório Carlos², Jaciara Bezerra Marques², Leika Aparecida Ishigama Geniole¹, Lia Márcia Cruz

da Silveira ${ }^{1}$, Maria Eugênia Bresolin Pinto², Nayra Almeida da Silva ${ }^{1}$, Rodrigo Cechelero Bagatelli', Sandro Rogério Rodrigues Batista², Tânia de Araújo Barboza1 ${ }^{1}$, Thiago Dias Sarti², Vitor Barreto', Gustavo Diniz Ferreira Gusso ${ }^{1}$, Mourad Ibrahim Belaciano ${ }^{2}$

Palavras-chave: Educação Médica Atenção Primária à Saúde Currículo

Keywords: Education, Medical Primary Health Care Curriculum

\section{Resumo}

0 presente documento traz diretrizes construídas conjuntamente pela Associação Brasileira de Educação Médica (ABEM) e a Sociedade Brasileira de Medicina de Família e Comunidade (SBMFC) com a intenção de apoiar as escolas médicas de forma objetiva e prática, na elaboração de projetos político-pedagógicos no contexto da Atenção Primária à Saúde. Um marco reconhecido na política educacional brasileira é a publicação das Diretrizes Curriculares Nacionais, as quais flexibilizam as organizações curriculares, possibilitando a construção de projetos político-pedagógicos contemporâneos e consonantes com o Sistema Único de Saúde brasileiro. A Atenção Primária à Saúde é o ponto de convergência entre estas duas políticas, descentralizando o ensino da Medicina dos hospitais para toda a rede de saúde no Brasil. Destaca-se a imperiosidade de que o ensino na Atenção Primária à Saúde esteja presente longitudinalmente, ao longo de todo o curso, de preferência com inserções significativas (de aprendizado real e a partir do trabalho), mas que, sobretudo, deva fazer parte do núcleo de ensino da prática clínica do futuro médico.

\section{Abstract}

These are a set of guidelines built by the Brazilian Association of Medical Education (ABEM) and the Brazilian Society of Family and Community Medicine (SBMFC) with the aim of supporting medical schools in a practical and objective manner, when elaborating pedagogical-political projects on Primary Health Care (PHC). The advent of the Brazilian National Curricular Guidelines for Medical

Sociedade Brasileira de Medicina de Família e Comunidade (SBMFC). demarzo@unifesp. br; thiagotrindade@terra com br; inezpadula@ yahoo.com.br; fabiano.guimaraes@globo.com; fepless@hotmail.com; doutor.flavio@ig.com.br; leikageniole@terra.com.br; lia.silveira@ uol.com.br; nayramed@hotmail.com; rodrigobagatelli@yahoo.com.br; tannia_shaktab@yahoo.com.br; barretovitor@hotmail.com; gustavo.gusso@gmail.com

${ }^{2}$ Associação Brasileira de Educação Médica (ABEM). rodrigocarii@yahoo.com.br; jinmarins@yahoo.com.br; astein@via-rs.net; proenco@ hotmail.com; arsego@via-rs.net; gustavotc@yahoo.com.br; eujaciara@gmail.com; eugenia@ufcspa.edu.br; sandrorbatista@gmail.com; thiagosarti@yahoo.com.br; mourad@saude.df.gov.br

Endereço para correspondência: Marcelo Demarzo - Departamento de Medicina Preventiva, Escola Paulista de Medicina, Universidade Federal de São Paulo, Rua Borges Lagoa, 1.341, Vila Clementino, São Paulo (SP), Brasil - CEP 04038-036.

Conflito de interesses: declararam não haver

Fonte de financiamento: ABEM e SBMFC.

Recebido em: 02/02/2011

Aprovado em: 26/02/2011 
Education, which are approved by the Ministry of Education in 2001 have since improved the teaching of undergraduate medical students on PHC, but there are still wide variations in implementation and quality of it in medical curricula. These guidelines by ABEM/SBMFC partnership can exert considerable influence on medical curricula by establishing minimum requirements and core competencies for PHC in Brazil.

\begin{abstract}
Palabras clave: Educación Médica Atención Primaria de Salud Curriculum
\end{abstract}

\section{Resumen}

Estos son estándares desarrollados conjuntamente por la Asociación Brasileña de Educación Medica (ABEM) y por la Sociedad Brasileña de Medicina Familiar y Comunitaria (SBMFC) destinados a ayudar las escuelas de medicina de una manera objetiva y práctica en el desarrollo de sus proyectos político-pedagógicos en el contexto de la Atención Primaria de la Salud. Un punto de referencia reconocido en la política educativa brasileña es la publicación de las Directrices Curriculares Nacionales, que traen la perspectiva de un currículo flexible, permitiendo la construcción de ellos de acuerdo con el Sistema Nacional de Salud en Brasil. La Atención Primaria de la Salud es un punto de convergencia entre estas dos políticas, la descentralización de la educación médica y de los servicios de los hospitales para la red de salud en su conjunto en el Brasil. Se destaca la necesidad urgente de que la educación en Atención Primaria de la Salud sea presente a lo largo de toda la formación de pregrado, de preferencia con inserciones significativas (aprendizaje real y desde el trabajo), pero, sobre todo, debe ser parte central de la enseñanza de la práctica clínica del futuro médico.

\section{Introdução}

Um marco reconhecido na política educacional brasileira é a publicação das Diretrizes Curriculares $\mathrm{Na}$ cionais $(\mathrm{DCN})^{1}$, as quais flexibilizam as organizações curriculares, possibilitando a construção de projetos político-pedagógicos (PPP) mais condizentes com o pensamento contemporâneo. A política de saúde no Brasil também experimenta um marco em sua história recente com a Constituição Federal de 1988, que instituiu em todo o território nacional um sistema universal e único de saúde, o Sistema Único de Saúde (SUS). Dentre inúmeras proposições contundentes, a Constituição definiu que a ordenação da formação de recursos humanos também é competência do SUS. A Atenção Primária à Saúde (APS) é ponto de convergência entre estas duas políticas, na medida em que as DCN apontam para uma integração com o sistema de saúde e com as necessidades de saúde da população, descentralizando o ensino da medicina dos hospitais à rede de saúde, tendo, na Estratégia de Saúde da Família (ESF), o modelo prioritário para a (re) organização da APS e toda a atenção à saúde no país.

Neste contexto, a Associação Brasileira de Educação Médica (ABEM) e a Sociedade Brasileira de Medicina de Família e Comunidade (SBMFC) vêm empreendendo esforços conjuntos de produção técnica e pedagógica para apoiar as escolas no processo de mudança (documentos disponíveis nos portais das entidades na Internet: http:// www.sbmfc.org.br e http://www.abem-educamed.org. br), com base nas DCN e com foco no ensino da APS na graduação em Medicina. Para a produção das presentes Diretrizes, as entidades examinaram documentos e publicações de pesquisadores nacionais das áreas de Saúde Coletiva e de Educação Médica, além de resoluções nacionais e internacionais para o ensino da APS e da Medicina de Família e Comunidade (MFC), que é entendida como especialidade médica com foco privilegiado na APS.

\section{Percurso metodológico}

Para construção das Diretrizes, inicialmente, elaborou-se um documento preliminar ${ }^{2}$ numa oficina de trabalho com duração de três dias, com professores e preceptores que atuam na APS e MFC, considerados espertos no tema pelas duas entidades. Com a finalização do documento preliminar em 26 de julho de 2009, iniciou-se uma consulta pública nos portais virtuais das duas entidades, como também nos congressos nacionais: Congresso Brasileiro de Educação Médica (COBEM), de 2009 e 2010, e Congresso Brasileiro de Medicina de Família e Comunidade, de 2009, garantindo assim participação ampla, coletiva e democrática de professores, preceptores, estudantes, gestores e pessoas da comunidade. O presente documento é a síntese do documento preliminar e das sugestões que apareceram na consulta pública, cujo encerramento ocorreu em 29 de outubro de 2010 durante o COBEM.

\section{Diretrizes}

Os resultados apresentados estão organizados como: contribuições da APS para a graduação em Medicina ("por quê"); objetos de aprendizado ("o quê"); metodologias e estratégias didáticas ("como"); momentos ("quando"); espaços formativos ("onde") e os principais atores envolvidos ("quem"). 


\section{"Por quê?" - contribuições da Atenção Primária à Saúde para a graduação em Medicina}

- O estudante é favorecido ao lidar com diferentes aspectos da vida e seus ciclos, na sua complexidade clínica e cultural.

- É possibilitada ao estudante a atuação em relação ao indivíduo e ao coletivo de forma contextualizada à realidade local.

- Constitui cenário de integração de práticas das diferentes áreas, campos e núcleos de conhecimento (ciências básicas, especialidades médicas e saúde coletiva).

- Contribui para o estudante ter uma compreensão da rede intersetorial de atenção e cuidados em saúde.

- Contribui para o desenvolvimento de uma prática clínica integrada, possibilitando a interdisciplinaridade.

- Favorece o aumento da resolubilidade clínica ao lidar com condições e problemas complexos e singulares de saúde, de forma contínua e longitudinal.

- Contribui para conceituação de saúde e adoecimento, respeitando o saber do outro e da comunidade local.

- Contribui para a concepção de conhecimento dinâmico e em construção, que articule outros conhecimentos e realidades.

- Favorece o desenvolvimento da competência cultural e dialógica na comunicação em saúde.

\section{"O quê?" - objetos de ensino- aprendizagem}

Entende-se que a ordem cronológica não interfere no desenvolvimento da competência final em APS, desde que as três abordagens constituam eixos estruturantes da formação médica e sejam desenvolvidas ao longo dos seis anos de curso. Ao final do sexto ano, o estudante de Medicina deve ter competência nas três dimensões a seguir, de forma integrada:

$\mathrm{Na}$ abordagem individual, deve-se:

- conhecer e utilizar a abordagem clínica centrada na pessoa integral, complexa, interdisciplinar, longitudinal e resolutiva, utilizando as evidências científicas como ferramenta e suporte, porém, singularizando o processo;

- estabelecer o primeiro contato com os pacientes, lidando com problemas não-selecionados e indiferenciados, reconhecendo as incertezas no cotidiano da prática clínica da APS;
- desenvolver e aplicar a consulta do médico de família e comunidade para promover uma eficaz relação médicopaciente, com respeito pela autonomia deste;

- relacionar os processos específicos de decisão com a prevalência e a incidência das doenças na comunidade;

- reunir e interpretar seletivamente a informação recolhida na anamnese, no exame objetivo e nos exames complementares, e aplicá-la a um plano de ação adequado em colaboração com o paciente;

- manejar simultaneamente múltiplas queixas e patologias, tanto problemas de saúde agudos como crônicos das pessoas;

- promover a saúde e o bem-estar, aplicando adequadamente as estratégias de promoção da saúde e prevenção da doença;

- conciliar as necessidades de cada paciente e as de saúde da comunidade em que ele vive, de acordo com os recursos disponíveis.

$\mathrm{Na}$ abordagem familiar, deve-se: conhecer e lidar com as distintas fases do ciclo vital; conhecer e lidar com a estrutura e dinâmica familiar, utilizando os instrumentos do diagnóstico familiar, como o genograma e ecomapa na abordagem familiar; identificar a influência das relações intrafamiliares no processo de saúde e adoecimento.

$\mathrm{Na}$ abordagem comunitária, deve-se: conhecer e lidar com instrumentos de diagnóstico de saúde da comunidade, acessando os diversos setores relacionados e correlacionando-os com a prática clínica do médico; identificar a organização da sociedade e da comunidade, os modos de produção presentes e os determinantes sociais do processo saúde-adoecimento; identificar e respeitar a diversidade cultural; compreender o que é "território vivo"; reconhecer e desenvolver ações de vigilância em saúde e participar de atividades de educação popular em saúde, compreendendo a existência de diferentes concepções pedagógicas e valorizando o saber popular.

\section{"Quando?" - momentos formativos}

Destacam-se as seguintes orientações gerais quanto aos tempos de inserção do ensino da APS nas grades curriculares de Medicina:

- deve ser uma inserção longitudinal e com continuidade ao longo do curso, num modelo em espiral crescente de complexidade e, de preferência, com atividades na APS em todos os períodos (semestre ou anos) do curso;

- devem ser incluídas, desde o primeiro ano, atividades envolvendo as abordagens do indivíduo, da família e da comunidade, de forma integrada; 
- devem ser introduzidos módulos, disciplinas, unidades educacionais ou estágios de APS centrados na clínica do médico de família (método clínico centrado na pessoa), nos ciclos e anos intermediários (terceiro e quarto anos e períodos correspondentes);

- as competências (conhecimentos, habilidades e atitudes) adquiridas ao longo dos anos na APS, incluindo os estágios práticos, devem ser mais bem sedimentadas durante o internato (quinto e sexto anos), em estágios de treinamento clínico em serviços de APS, sob supervisão.

\section{"Como?" - diretrizes metodológicas e estratégias didáticas}

Como diretrizes metodológicas e estratégias didáticas, são fundamentais:

- inserção significativa, com objetivos claros de ensinoaprendizagem e cronograma de trabalho, evitando-se apenas estágios de observação; com estratégias que impliquem o estudante na corresponsabilização do cuidado, contribuindo efetivamente para o serviço em que ocorre a prática, construindo projetos terapêuticos em conjunto nas equipes de APS dentro de cada unidade;

- metodologias dialógicas e ativas de ensino-aprendizagem como referência no ensino na APS, integrando prática e teoria, problematizando a realidade e possibilitando a reflexão sobre a prática profissional, trabalhando em pequenos grupos de forma espiral (progressiva) e com diversidade de cenários e atividades;

- favorecimento de ambiente multi e interdisciplinar e atuação conjunta com estudantes e profissionais de outras áreas;

- inserção no contexto do desenvolvimento das habilidades clínicas, com foco no seguimento de pessoas e famílias, desde o início da graduação;

- integração com outras disciplinas do curso de Medicina, possibilitando espaços de troca, tanto ao longo do curso como num semestre específico;

- estímulo para a utilização adequada e contextualizada de condutas clínicas baseadas em evidências científicas, estimulando e aproximando o estudante da investigação e produção científica; ao mesmo tempo, singularizando o processo de produção do cuidado, com foco nas pessoas e comunidades;

- pactuação conjunta com as equipes de APS, gestor local e com a comunidade das atividades e ações individuais e coletivas a serem realizadas pelos estudantes;
- incentivo para a utilização de estratégias de ensino à distância e telessaúde;

- busca de integração com pesquisa e extensão;

- utilização de métodos avaliativos formativos, além dos somativos, os quais sejam contextualizados e adequados ao desenvolvimento da competência em APS.

\section{“Onde?" - espaços formativos}

Entende-se, como cenários ou equipamentos de APS adequados enquanto espaços formativos para a graduação em Medicina unidades, equipamentos de saúde e redes de apoio social próprios de APS, incluindo o sistema suplementar, desde que possibilitem o desenvolvimento das competências definidas nestas diretrizes, dentro da lógica definida atualmente pela Política Nacional de Atenção Básica $(\mathrm{PNAB})^{3}$, com estrutura adequada para receber atividades de ensino e estudantes; a estrutura adequada deve ser pactuada entre os atores da integração ensino-serviçogestão e comunidade, com base nas diretrizes de qualidade do Ministério da Saúde e da Educação, levando-se em consideração:

- a existência de um número e tamanho adequados de salas de atendimento individual e coletivo para participação efetiva dos estudantes nas atividades da equipe de APS;

- a existência de espaço físico de estudo e conforto para os estudantes, com acesso ao material bibliográfico próprio da APS;

- a existência de meio de transporte público que permita o acesso adequado dos estudantes às unidades e aos equipamentos de saúde;

- o estabelecimento de instrumentos jurídico-legais firmados entre as Instituições de Ensino Superior (IES) e o gestor municipal, os quais estabeleçam o desenvolvimento de atividades em longo prazo, com a inclusão de colegiados gestores paritários, tendo a participação de estudantes e do controle social;

- Unidades de Saúde da Família ou similares, integradas à rede municipal de saúde, articuladas em todos os níveis do sistema, com estímulo para que as IES participem no desenvolvimento dessa rede;

- preferencialmente, concentrando as atividades em determinado território ou distrito, a fim de permitir melhor acompanhamento, continuidade e contextualização do desenvolvimento das atividades, integrando os diversos equipamentos da rede de atenção à saúde;

- preferencialmente em Unidades de Saúde que tenham Programas de Residência em MFC; 
- utilizando equipamentos comunitários enquanto espaços de formação.

\section{"Quem?" - docentes e preceptores}

Considerando-se o acúmulo internacional sobre a participação do médico especialista em MFC no ensino da APS, na graduação em Medicina, e respeitando as necessidades e a autonomia de cada IES, é fundamental a participação do médico especialista em MFC (com residência médica ou título de especialista na área) no quadro de docentes e preceptores. Recomenda-se ter uma proporção adequada de docentes especialistas em MFC entre os docentes universitários, para adequar-se ao modelo pedagógico e às necessidades de saúde da população.

Também devem ser criadas vagas docentes, com carga horária adequada, dentro da estrutura universitária de cada IES, as quais garantam a presença do médico especialista em MFC e dos demais profissionais da APS.

O ensino da abordagem clínica individual na APS, principalmente nos anos intermediários do curso e no internato, deve ser realizado pelo médico especialista em MFC, em parceria com outros profissionais clínicos com formação e/ ou vivência em tal cenário. A Figura 1 traz uma representação gráfica em " $Z$ ”, sugerindo a referência docente para o estudante ao longo dos seis anos do curso médico, no cenário da APS.

$\mathrm{Na}$ abordagem familiar e comunitária, deve haver, além do médico especialista em MFC, outros profissionais com competência em tais abordagens.

Caso não exista no quadro da IES o especialista em MFC, a instituição deve pactuar com os gestores universitários e do sistema de saúde a formação e/ou especialização deste profissional médico.

Deve haver um programa de desenvolvimento profissional contínuo e uma educação permanente para a docência em APS, incluindo docentes e médicos dos serviços (preceptores), desenvolvido pelas IES, em parceria com o gestor local de saúde. O docente da IES deve estar efetivamente inserido nas equipes de APS para acompanhar e supervisionar o processo.

É bom haver uma ótima relação entre o número de preceptores e estudantes em cada equipe de APS, pensando na qualidade da preceptoria.

Deve-se garantir que a agenda de atividades do preceptor nas unidades de saúde seja compatível com as atividades de ensino, assistência e educação permanente. $\mathrm{O}$ Quadro 1 sumariza as principais recomendações das Diretrizes.
Figura 1. Representação esquemática em "Z" da referência docente para 0 estudante no cenário da Atenção Primária à Saúde, ao longo dos seis anos do curso médico.

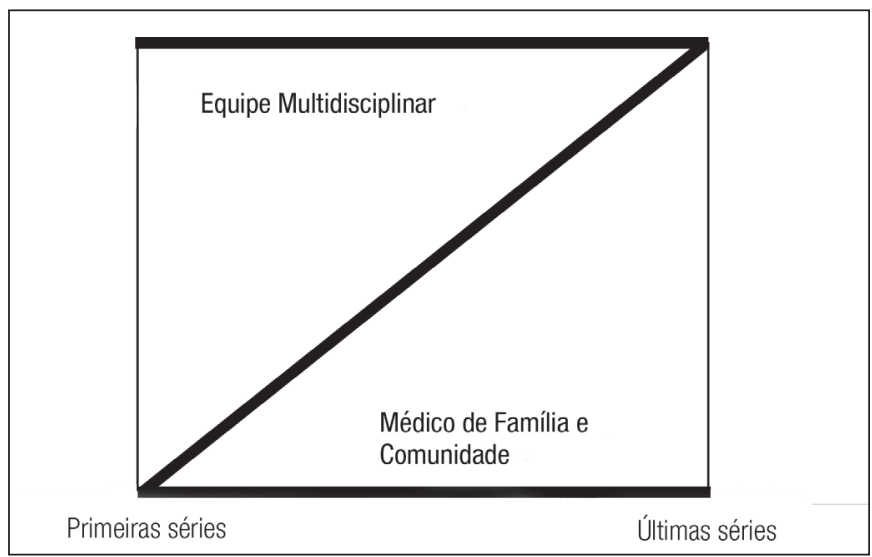

Quadro 1. Sumário das diretrizes para o ensino na APS na graduação em Medicina.

\begin{tabular}{|l|}
\hline Por que ensinar na APS? \\
\hline - Contribui para o desenvolvimento de uma prática clínica integrada e \\
contextualizada, que é centrada nas pessoas e comunidades, possibilitando a \\
interdisciplinaridade.
\end{tabular}

\section{Considerações finais}

Este é um documento "vivo", que deve estar em constante processo de atualização e revisão, e que visa, sobretudo, desencadear as discussões sobre o tema. Trata-se de diretrizes construídas com a intenção de apoiar as escolas médicas, de forma objetiva e prática, na elaboração de seus projetos pedagógicos no contexto da APS.

Considera-se que os núcleos do saber e do agir médico se encontram no aprendizado da clínica, mais especificamente no raciocínio clínico e semiológico. Por isso, 
destaca-se a imperiosidade de que o ensino na APS esteja presente longitudinalmente ao longo do curso, de preferência com inserções significativas (de aprendizado real e a partir do trabalho), mas que, sobretudo, deva fazer parte do núcleo do ensino da semiologia e da prática clínica do futuro médico.

Enfim, tais Diretrizes apontam para a necessidade de outros processos institucionais e políticos nas áreas de educação e saúde para a sua efetivação. Faz-se necessário uma agenda junto aos Ministérios da Saúde e da Educação, como também junto às IES, para que sejam discutidas estratégias de ampliação e renovação do quadro de docentes no Ensino Superior, assim como de qualificação dos serviços e equipamentos de APS, expressos em contratos claros de compartilhamento dos objetos de gestão e ensino, contribuindo para a melhora das condições da graduação em Medicina no Brasil.

\section{Agradecimentos}

A todos os participantes da consulta pública nos portais virtuais e nos congressos da ABEM e SBMFC, pela disposição e empenho na elaboração destas Diretrizes.

\section{Referências}

1. Brasil. Ministério da Educação. Conselho Nacional de Educação. Câmara de Educação Superior. Resolução CNE/ CES n 4, de 07 de novembro de 2001. Diretrizes Curriculares Nacionais do Curso de Graduação em Medicina. [online]. Brasília, DF; 2001. [capturado 1 nov. 2010]. Disponível em: http://portal.mec.gov.br/cne/arquivos/pdf/CES04.pdf

2. Gusso G, Marins JJN, Demarzo MMP, Belaciano Ml, Almeida RCC, Stein AT, et al. Diretrizes para o Ensino na Atenção Primária à Saúde na Graduação em Medicina - SBMFC e ABEM. Cad ABEM. 2009 [online]; 5: 13-20. [capturado 1 nov. 2010]. Disponível em: http://www.abem-educmed.org.br/pdf caderno5/atencao primaria saude.pdf

3. Brasil. Ministério da Saúde. Departamento de Atenção Básica. Política Nacional de Atenção Básica. Brasília: Ministério da Saúde; 2006. 